\title{
Emprego de MER e CRE em Poisson 1D para análise do erro de variáveis secundárias
}

\author{
Ana Eliza Gonçalves Ferreira \\ Programa de pós-graduação em Engenharia Mecânica, UFPR, Curitiba, PR, \\ Departamento de Engenharia Mecânica. \\ Carlos Henrique Marchi ${ }^{2}$ \\ Departamento de Engenharia Mecânica, UFPR, Curitiba, PR
}

\begin{abstract}
Resumo. No presente trabalho, foi estudado a redução do erro de discretização na solução da equação de Poisson 1D. Analisou-se a influência que a redução do erro nas soluções nodais pode exercer sobre os erros do cálculo das variáveis secundárias temperatura média e inclinação em $x=0$. Para a redução do erro de discretização nas soluções nodais, foram empregados os métodos MER e CRE. O decaimento do erro a cada refino com extrapolação, ou sem extrapolação, foi acompanhado pela média da norma do erro dado pela diferença entra a solução numérica e analítica. Foram observadas as ordens efetivas dos erros de discretização em todas as variáveis. Os resultados obtidos indicam que com soluções nodais mais acuradas se confere, no máximo, a acurácia de cada método empregado na determinação das variáveis secundárias. Concluiu-se que as soluções nodais precisam ter ordem de acurácia igual ou superior às ordens de acurácia dos métodos utilizados para cálculo das variáveis secundárias para que se atinjam suas ordens teóricas. Nesse sentido, o emprego de MER e também de CRE se mostraram eficientes para melhorar a acurácia das soluções nodais e assim se obter a ordem teórica de acurácia dos métodos utilizados no cálculo de variáveis secundárias.
\end{abstract}

Palavras-chave. Multiextrapolação de Richardson, Extrapolação de Richardson Completa, Erro de discretização, Variáveis Secundárias

\section{Introdução}

Os erros numéricos podem ocorrer por influência da discretização das equações diferenciais, do arredondamento das operações, das iterações e da programação empregada para a solução. Por meio de verificação e validação $(V \& V)$ de soluções para um campo de velocidades em um problema de Dinâmica de Fluidos Computacional (CFD), sabe-se que o erro de discretização $(E h)$ representa a principal fonte de erro numérico [2].

\footnotetext{
1 anaeliza_gf@hotmail.com
}

2 chmcfd@gmail.com 
Foi utilizada, neste trabalho, a equação de Poisson 1D, Eq. 1, resolvida pelo Método de Diferenças Finitas (MDF) [1], em malha uniforme e com o termo fonte $f(x)=e^{x}$,

$$
\frac{d T^{2}}{d x^{2}}=f(x), 0 \leq x \leq 1 .
$$

Foram empregados os métodos de Multiextrapolação de Richardson (MER) [8] e Extrapolação de Richardson Completa (CRE) [9]. Observou-se como a redução do erro, que estes métodos conferem às soluções nodais, impacta no erro da solução de variáveis secundárias como temperatura média e inclinação em $x=0$. Ambos os métodos, para redução do erro de discretização, já foram comprovados na literatura para soluções nodais. Para a resolução de um problema de condução de calor governado pela equação de Laplace 2D, MER reduz significativamente o erro de discretização [6]. Já o emprego de MER na resolução numérica pelo Método de Volumes Finitos (MVF) da equação de Laplace 2D para até onze níveis de extrapolação e se constata que a redução do erro numérico é dependente da variável de interesse e da geometria de cálculo [7]. Por sua vez, em testes com CRE nas equações de Poisson, advecção-difusão, Laplace e Burgers pelo método de diferenças finitas, com aproximações de $1^{\mathrm{a}}, 2^{\mathrm{a}}$ e $4^{\mathrm{a}}$ ordens de acurácia se verifica que reduz o erro de discretização de soluções [4].

Neste trabalho, a seção 2 apresenta a metodologia, com as implementações que foram feitas para produzir os resultados. A seção 3 aborda os experimentos computacionais realizados e apresenta os resultados obtidos por meio de gráficos. A seção 4 expõe as conclusões e, por fim, fazem-se agradecimentos e apresentam-se as referências utilizadas.

\section{Metodologia}

A Eq. 1, para resolução por MDF, foi discretizada em malha uniforme, representada na Figura 1,

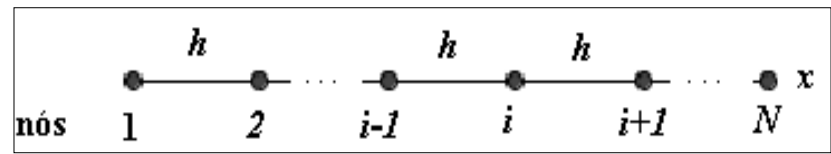

Figura 1: Representação de uma malha unidimensional uniforme com $N$ nós

com a utilização da aproximação CDS-2 (Central Difference Scheme de segunda ordem), Eq. 2,

$$
\frac{d T^{2}}{d x^{2}} \approx \frac{T\left(x_{i-1}\right)+T\left(x_{i+1}\right)-2 T\left(x_{i}\right)}{h^{2}},
$$

onde $h$ é o tamanho dos elementos de malha, isto é $h=1 /(N-1)$ e $N$ o número de nós.

Quanto maior o número de pontos utilizados numa aproximação, maior o risco de instabilidade do método numérico [3]. A utilização do esquema CDS-2 se torna interessante por ser de $2^{a}$ ordem de acurácia e envolver somente dois pontos nodais [5].

O sistema linear resultante foi resolvido com o método TDMA (TriDiagonal Matrix Algorithm) [10] para malhas de $N=3$ nós a $N=1.048 .577$ nós e razão de refino $r=2$. A variável secundária temperatura média $(\bar{T})$ foi calculada numericamente com a Regra 
do Trapézio, Eq. 3, e com a Regra de Simpson, Eq. 4,

$$
\begin{gathered}
\overline{T_{T}}=\frac{h}{2} \sum_{i=2}^{N}\left(T\left(x_{i-1}\right)+T\left(x_{i}\right)\right) \\
\overline{T_{S}}=\frac{h}{3} \sum_{i=2,4,6, \ldots}^{N}\left(T\left(x_{i-1}\right)+4 T\left(x_{i}\right)+T\left(x_{i+1}\right)\right)
\end{gathered}
$$

onde $N$ é o número de nós da malha unidimensional.

A variável secundária inclinação em $x=0$ foi determinada a partir da discretização com os esquemas DDS (Dowstream difference scheme) de primeira (DDS-1), segunda (DDS-2) e terceira ordem (DDS-3), conforme a Eq. 5, Eq. 6 e Eq. 7 respectivamente.

$$
\begin{gathered}
\left(\frac{d T}{d x}\right)_{x=0}=\frac{T(2)-T(1)}{h} \\
\left(\frac{d T}{d x}\right)_{x=0}=\frac{-3 T(1)+4 T(2)-T(3)}{2 h} \\
\left(\frac{d T}{d x}\right)_{x=0}=\frac{-11 T(1)+18 T(2)-9 T(3)+2 T(4)}{6 h}
\end{gathered}
$$

Em pós-processamento foi empregado MER cuja expressão é dada pela Eq. 8 que, do ponto de vista teórico, pode ser repetida infinitamente,

$$
\phi_{g, m}=\phi_{g, m-1}+\frac{\phi_{g, m-1}-\phi_{g-1, m-1}}{r^{P L-1}-1}
$$

onde $\phi$ é a solução numérica da variável de interesse, $m$ é o nível de extrapolação, $P L$ é a ordem assintótica do erro e $g$ é o número de malhas.

Foi calculado também CRE, onde se utiliza a solução de todos os nós da malha, ou seja, de todo o campo do domínio de cálculo da Eq. 1. Para os nós ímpares, a CRE é empregada conforme a Eq. 9,

$$
\phi_{g, 1}^{i}=\phi_{g, 0}^{i}+\operatorname{Tcorr}_{g, 0}^{i}
$$

onde $i$ é a posição do nó e o termo de correção é dado pela Eq. 10,

$$
\operatorname{Tcorr}_{g, 0}^{i}=\frac{\phi_{g, 0}^{i}-\phi_{g-1,0}^{i}}{r^{P L}-1}
$$

O termo de correção é o estimador de Richardson baseado na ordem assintótica. Para os nós pares, a expressão de CRE é mostrada na Eq. 11,

$$
\phi_{g, 1}^{i+1}=\phi_{g, 0}^{i+1}+\operatorname{Tcorr}_{g, 0}^{i+1}
$$

e o termo de correção é dado pela média dos termos de correção calculados para os nós ímpares conforme Eq. 12,

$$
\operatorname{Tcorr}_{g, 0}^{i+1}=\frac{\operatorname{Tcorr}_{g, 0}^{i}+\operatorname{Tcorr}_{g, 0}^{i+2}}{2}
$$


O acompanhamento do decaimento do erro a cada refino com extrapolação, ou sem extrapolação, pode ser acompanhado pela média da norma do erro dado pela diferença entra a solução numérica e analítica $(\overline{L 1})$, dada pela Eq. 13 ,

$$
\overline{L 1}=\frac{1}{N-1} \sum_{i=2}^{N-1}\left(T\left(x_{i}\right)-T a\left(x_{i}\right)\right)
$$

onde $T$ é a temperatura numérica, $T a$ é a temperatura analítica e $N$ é o número de nós da malha, incluindo os contornos.

\section{Experimentos Computacionais}

Os códigos computacionais, dos métodos descritos na seção 2, foram implementados em linguagem Fortran 90, com precisão quádrupla, e executados em um microcomputador de CPU Intel(R) Core(TM)2, $2.66 \mathrm{GHz}$ e 8GB de RAM com sistema operacional Windows XP 2003 de 64bits.

O comportamento dos erros da temperatura média e da inclinação em $x=0$, com a solução sem extrapolação e sob emprego de MER e CRE pode ser observado na Figura 2. Com o emprego de MER é possível observar que o erro nas soluções nodais da Eq. 1 diminui a cada nível de extrapolação. Analogamente, o efeito de CRE nas soluções nodais é de aumentar a ordem de acurácia das soluções nodais.

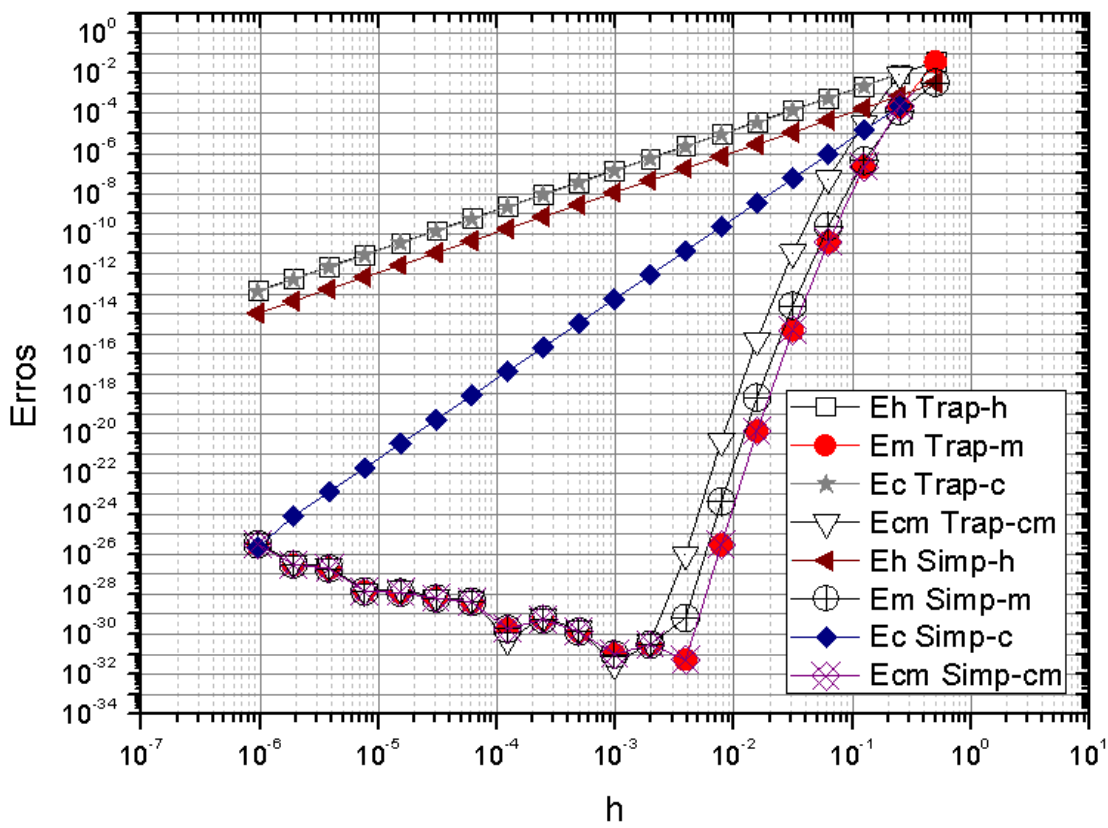

Figura 2: Erros da temperatura média com Regras do Trapézio e Simpson, sem extrapolação e extrapoladas com MER e CRE.

Na Figura 2 temos que Eh Trap-h representa o erro da temperatura média com a Regra 
do Trapézio sem extrapolação; Em Trap-m, o erro da Regra do Trapézio com MER; Ec Trap-c, o erro da Regra do Trapézio com CRE; Ecm Trap cm, o erro da Regra Trapézio com MER sobre CRE. De maneira análoga temos para a Regra de Simpson.

A ordem efetiva equivalente das curvas da Figura 2, para as Regras do Trapézio e de Simpson sem extrapolação resultaram em 2. Após o emprego de CRE na temperatura média pela Regra do Trapézio a ordem efetiva equivalente não se altera e com a Regra de Simpson resultou em 4. Com MER, os erros diminuem e as ordens efetivas equivalentes aumentam, até no máximo 19 na $6^{\mathrm{a}}$ extrapolação.

A Regra do Trapézio possui ordem de acurácia teórica 2 e a Regra de Simpson ordem 4. Nota-se que com melhores resultados nodais, a maior ordem atingida é a ordem teórica do método de cálculo da variável secundária quando se aplica CRE. Contudo, MER se mostra eficiente para atuar diretamente no decaimento dos erros e aumento da ordem de acurácia independente da ordem de acurácia do método empregado no cálculo das variáveis secundárias.

A variável secundária inclinação em $x=0$ foi determinada com esquemas de ordem de acurácia 1 (DDS-1), 2 (DDS-2) e 3 (DDS-3). Sem o emprego das extrapolações, as ordens efetivas foram 1, 2 e 2, respectivamente. Após as extrapolações com MER e com CRE ocorreu o que se pode ver na Figura 2. Após o emprego de CRE, os resultados indicam que o erro atingiu a ordem teórica de cada esquema utilizado.

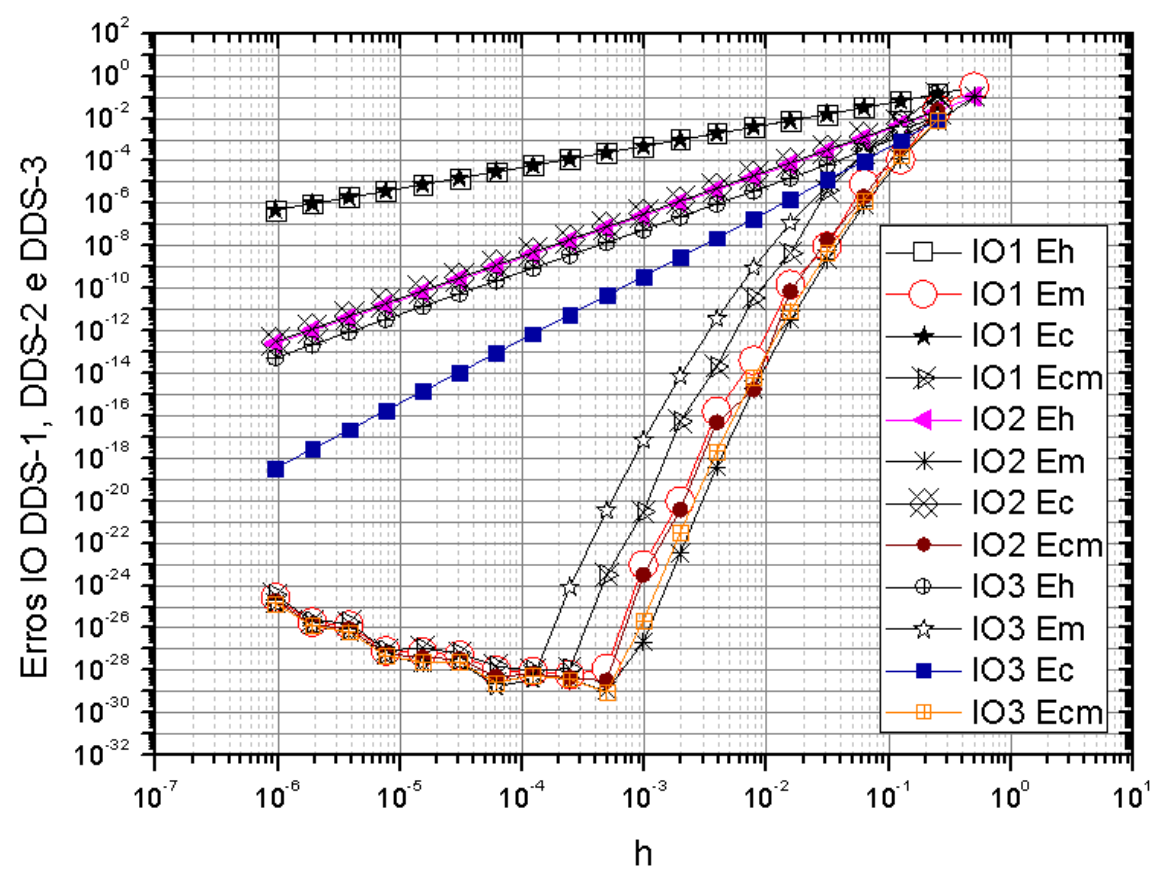

Figura 3: Erros da inclinação em $x=0$, com esquemas DDS-1 (IO1), DDS-2 (IO2) e DDS-3 (IO3), sem extrapolação e extrapoladas com MER e CRE.

Na Figura 3 temos IO1 Eh, o erro da inclinação em $x=0$ com DDS- 1 sem extrapolação; IO1 Em, o erro de DDS-1 com MER; IO1 Ec, o erro de DDS-1 com CRE; IO1 Ecm, o erro de DDS-1 com MER sobre CRE. Analogamente temos com uso de DDS-2 e DDS-3. A ordem efetiva equivalente das curvas da Figura 3, para o esquema DDS-1 sem extrapolação 
resultou 1 e para DDS-2 e DDS-3 resultaram 2. Após o emprego de CRE, a ordem efetiva equivalente não se alterou para DDS-1 e DDS-2 e para DDS-3 resultou 3.

Com MER, os erros diminuem e as ordens efetivas equivalentes aumentam, tendo apresentado melhor desempenho com DDS-3 para MER sobre CRE, atingindo 14 na $8^{\mathrm{a}}$ extrapolação. $\mathrm{O}$ emprego de CRE resultou em soluções nodais mais acuradas, como podemos observar pela redução da média da norma L1 do erro mostrada na Figura 4. O decaimento da média da norma L1 fica evidente pelo aumento da ordem efetiva equivalente que, sem extrapolação, resultou 2 e, após emprego de CRE, resultou 4.

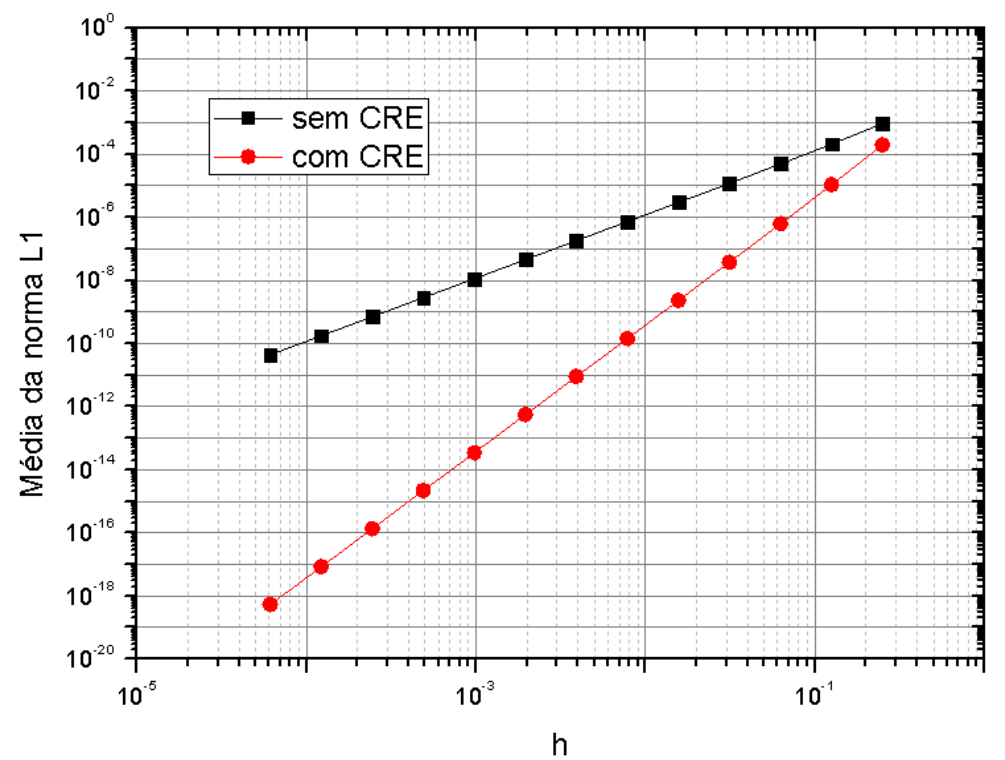

Figura 4: Média da norma do erro em 20 malhas sem extrapolação e com CRE.

\section{Conclusões}

Com o emprego de CRE foi possível obter a ordem teórica dos métodos mais acurados aplicados para as variáveis secundárias, Regra de Simpson para a temperatura média e esquema DDS-3 para a inclinação em $x=0$.

Os resultados obtidos neste trabalho indicam que soluções nodais mais acuradas são limitadas pelas acurácias dos métodos de determinação das variáveis secundárias. No início da resolução, para a discretização da Eq. 1, foi empregado o esquema CDS-2 que possui ordem 2 de acurácia. Isso influenciou as soluções sem extrapolação das variáveis secundárias, onde a máxima acurácia ficou limitada em 2, mesmo para o esquema DDS3 com ordem 3 para a inclinação em $x=0$ e para a Regra da Simpson com ordem 4 para a integração e obtenção da temperatura média.

Assim, as soluções nodais precisam ter ordem de acurácia igual ou superior às ordens dos métodos utilizados no cálculo das variáveis secundárias para que se atinjam suas ordens teóricas. Nesse sentido, o emprego de MER e também de CRE se mostram eficientes para, a partir da melhora da acurácia de soluções nodais, obter-se a ordem teórica de acurácia dos métodos utilizados no cálculo de variáveis secundárias. 


\section{Agradecimentos}

Agradecemos ao programa de pós-graduação em Engenharia Mecânica da UFPR; à infraestrutura física e computacional da UFPR, especialmente o laboratório de experimentação numérica Lena-1; à Capes, pela bolsa de doutorado da primeira autora e ao CNPQ pela bolsa do segundo autor.

\section{Referências}

[1] S. C. Chapra e R. P. Canale, Métodos Numéricos para Engenharia. 5 ed. Tradução: Helena Castro, McGraw-Hill, (2008).

[2] T. Castiglione, F. Stern, S. Bova and M. Kandasamy, Numerical investigation of the seakeeping behavior of a catamaran advancing in regular head waves, Ocean Engineering, v.38, p. 1806-1822, (2011).

[3] A. O. Fortuna, Técnicas Computacionais para Dinâmica dos Fluidos, Edusp, (2000).

[4] F. de F. Giacomini, C. H. Marchi e C. D. Santiago, Multiextrapolação de Richardson para reduzir erro de discretização em campos de CFD, Anais do Congresso de Matemática Aplicada e Computacional, (2013).

[5] C. R. Maliska, Transferência de calor e mecânica dos fluidos computacional. $2^{a}$ ed., LTC, (2004).

[6] C. H. Marchi, L. A. Novak e C. D. Santiago, Múltiplas extrapolações de Richardson para reduzir e estimar o erro de discretização da Equação de Laplace 2D, Proceeding Series of the Iberian Latin American Congress on the Computational Methods in Engineering, (2008).

[7] C. H. Marchi, L. K. Araki, A. C. Alves, R. Suero, S. F. T. Gonçalves and M. A. V. Pinto, Repeated Richardson extrapolation applied to the two-dimensional Laplace equation using triangular and square grids, Applied Mathematical Modelling, v. 37, n.1, p. $4661-4675,(2013)$.

[8] L. F. Richardson and J. A. Gaunt, The Deferred Approach to the Limit, Phylosophical Proceedings of the Royal Society of London Serial A, v. 226, p. 299-361, (1927).

[9] P. J. Roache and P. M. Knupp, Completed Richardson Extrapolation, Communications in Numerical Methods in Engineering, v. 9, p. 365-374, (1993).

[10] H. K. Versteeg and W. Malalasekera, An introduction to computacional fuid dynamics, the finite volume method, 2 ed., Pearson/Prentice Hall, (2007). 